Cahiers $d u$ MONDE RUSSE

\section{Cahiers du monde russe}

Russie - Empire russe - Union soviétique et États indépendants

$43 / 4 \mid 2002$

Intellectuels et intelligentsia

\title{
Marshall T. Poe, A people born to slavery
}

\section{Pierre GONNEAU}

\section{OpenEdition \\ Journals}

Édition électronique

URL : https://journals.openedition.org/monderusse/1147

DOI : 10.4000/monderusse. 1147

ISSN : $1777-5388$

\section{Éditeur}

Éditions de l'EHESS

\section{Édition imprimée}

Date de publication : 30 décembre 2002

Pagination : $677-680$

ISBN : 2-7132-1796-2

ISSN : $1252-6576$

\section{Référence électronique}

Pierre GONNEAU, «Marshall T. Poe, A people born to slavery », Cahiers du monde russe [En ligne], 43/4 | 2002, mis en ligne le 17 juin 2009, consulté le 03 septembre 2022. URL : http://

journals.openedition.org/monderusse/1147; DOI : https://doi.org/10.4000/monderusse. 1147

Ce document a été généré automatiquement le 3 septembre 2022

Tous droits réservés 


\title{
Marshall T. Poe, A people born to slavery
}

\author{
Pierre GONNEAU
}

\section{RÉFÉRENCE}

Marshall T. POE, A people born to slavery. Russia in early modern European

ethnography, 1476-1748. Ithaca-Londres, Cornell University Press, 2000, 293 p. (Studies of the Harriman Institute)

1 Après nous avoir donné une importante bibliographie des sources étrangères concernant la Russie (Foreign descriptions of Muscovy : an analytic bibliography of primary and secondary sources, Columbus, 1993), Marshall Poe entreprend d'exploiter ce matériau. Son but est de " mettre en lumière les origines de l'image moderne de la Russie en analysant les récits européens consacrés à la Moscovie, écrits de la fin du XV siècle au début du XVIII ${ }^{\mathrm{e}}$. Sans prétendre à l'exhaustivité, il s'intéresse à «la genèse de cette idée simple et fondamentale selon laquelle le tsar était un tyran régnant sur des sujets esclaves » (p. 4). La plus grande attention est donc accordée aux témoignages concernant le gouvernement de la Russie d'une manière générale et les rapports entre le souverain et les élites en particulier. L'auteur est conscient que les textes qu'il utilise expriment, eux aussi, les idées d'une élite de voyageurs, de penseurs et de gouvernants plutôt qu'une véritable opinion publique européenne ; pourtant, ils contribuent largement à écrire une sorte de dictionnaire des idées reçues sur la Russie, appelées à une longue vie. Le sujet est, on le voit, important, et l'exposé, clair et agréablement rédigé, fournit d'intéressants éléments de réponse.

2 Les textes retenus sont classés en fonction de leur date de parution et de la qualité des informations qu'ils contiennent. Le chapitre 1 rend compte des seize descriptions de la Russie composées entre 1486 et 1549, toutes de seconde main. Le chapitre 2 est consacré aux récits de diplomates et de marchands qui firent en Moscovie des séjours de courte durée (deux ans au plus) entre 1549 et 1700. Le chapitre 3 traite des témoignages d'étrangers qui 
résidèrent plusieurs années dans le pays, à la même époque. Pour ces deux sections, Poe n'a retenu qu'une trentaine d'œuvres, les plus riches à ses yeux, sur un total nettement plus élevé (et non précisé). Les chapitres 4 à 6 entendent présenter la place qu'occupe la Russie dans cinq importants traités théoriques, composés entre 1576 et 1748 . Toutefois, comme leurs réflexions sont largement tributaires de l'ouvrage clé de Sigismond von Herberstein, Rerum moscoviticarum commentarii ( $1^{\mathrm{e}}$ édition: 1549), un important retour en arrière lui est consacré. Le chapitre 7 s'efforce d'établir dans quelle mesure la perception européenne correspondait aux réalités russes en se demandant si la Moscovie était un système despotique. On trouve encore en annexe une présentation des « histoires folkloriques » sur Ivan le Terrible contenues dans les sources utilisées. L'ouvrage est complété par une bibliographie conséquente, mais omettant les travaux français, ou italiens, comme il est souvent d'usage aux États-Unis ${ }^{1}$.

3 À la fin de l'introduction (p. 5-7), Poe précise les choix terminologiques qu'il a été amené à faire, quitte à commettre certains anachronismes. C'est ainsi qu'il distingue des textes cosmographiques, historiques, diplomatiques (rapports d'ambassade) et " ethnographiques ». Par ce dernier terme, il faut entendre les descriptions générales de la Russie qui, le plus souvent, comprennent les rubriques suivantes : «la géographie, la cour, l'administration, l'armée, les classes sociales, les mœurs et coutumes, l'économie, la religion, etc. ». Cette définition n'est guère choquante, mais il est étrange de voir les auteurs des $\mathrm{XVI}^{e}$ et $\mathrm{XVII}^{\mathrm{e}}$ siècles se transformer en "ethnographes", ou Montesquieu défini comme un « lecteur assidu de littérature ethnographique » (p. 192)...

4 L'auteur utilise également les termes « Russie » et "Russes » plutôt que « Moscovie » et "Moscovites»; il nomme ses observateurs étrangers des " Européens» et leur espace "l'Europe ». Ce parti pris a l'avantage d'éviter les "Occidentaux» et «l'Ouest», concepts inexistants avant le XIX ${ }^{e}$ siècle. En revanche, il présente l'inconvénient de renforcer la dichotomie entre Europe et Russie, alors que - le contenu du livre le montre - l'origine et les intérêts nationaux de chaque auteur influent parfois nettement sur sa peinture de la Russie. Mais il est aussi vrai, comme le prouvent les tableaux des p. 27, 37, 136 et 137, que les emprunts d'un ouvrage à l'autre étaient monnaie courante et que la vision d'Herberstein toucha, directement ou indirectement, l'ensemble de l'Europe. Cette observation se vérifie plus particulièrement en ce qui concerne le cœur de la problématique, la question du despotisme. "Quand les ethnographes utilisent Herberstein (ou quelque texte herbersteinien), l'image du despotisme tend à être présente. Des douze auteurs qui citent soit Herberstein, soit un texte qui emprunte à Herberstein, onze [...] mentionnent au moins trois des caractéristiques de la thèse despotique ». En revanche, « les ethnographes qui ne connaissent pas Herberstein passent sous silence l'interprétation despotique ou lui accordent peu d'attention» (p. 140-141). Même si l'histoire ne s'écrit pas avec des «si ", Poe touche un point important en s'interrogeant sur le destin qu'aurait pu avoir l'image de la Russie en Europe si l'Anglais Chancellor, laudateur du système politique russe, avait précédé Herberstein en Russie et pris la peine d'écrire une relation élaborée, en latin, plutôt qu'un obscur livret en anglais (p. 144). Mais la popularité d'Herberstein est-elle accidentelle, ou bien l'ambassadeur n'at-il pas écrit ce que le public éduqué européen était prêt à entendre sur la lointaine Russie?

5 La conclusion du dernier chapitre, sur la réalité du despotisme moscovite, est à la fois équilibrée et quelque peu décevante (p. 225-226). Tout en reconnaissant le caractère artificiel de l'opposition "binaire mythique " entre une Moscovie servile et une 
Europe libre, il considère que l'autorité du souverain moscovite dépassait celle de tout autre monarque européen. Marshall Poe admet aussi que les observateurs étrangers ont pris trop au pied de la lettre la terminologie des relations de maitre à esclave couramment employée à la cour de Moscou, sans voir que si le souverain/maître exigeait d'être servi, il s'engageait aussi à protéger le noble/esclave. Mais on peut dire que le même contrat existait entre le maître noble et son serf, explicitement formulé dans le cas où une charte d'asservissement volontaire (kabala) était rédigée. Selon Poe, la principale différence entre Russie et Europe se situe au niveau de la philosophie ou de la théologie politique: "Contrairement aux Européens, qui croyaient que le gouvernement devait être limité afin que les hommes puissent se perfectionner, les Moscovites pensaient que l'autorité du tsar devait être presque illimitée, afin de réfréner les penchants mauvais des hommes ". L'auteur estime enfin que le système autoritaire mis en place s'avérait efficace en ce qu'il évitait les querelles politiques au sommet (sic) et permettait de mobiliser d'importantes ressources fiscales à la base. Il ne s'agissait donc pas d'une tyrannie, mais plutôt "d'une stratégie logique d'adaptation qui permettait à l'élite moscovite de construire un empire dans des conditions des plus délicates ».

Nous ne partageons sans réserve que la remarque sur la fonction métaphorique du discours de la servitude. En ce qui concerne le despotisme, l'auteur n'a, visiblement, pas eu le temps de lire le livre de Donald Ostrowski, Muscovy and the Mongols : cross-cultural influences on the steppe frontier, 1304-1589, Cambridge, 1998, qui consacre son chapitre 4 au « despotisme oriental » de la Moscovie. Si l'analyse du système russe à laquelle se livre Ostrowski est discutable sur bien des points, cet auteur nous semble proche de la vérité lorsqu'il conclut que despotisme et servilité sont des catégories élaborées d'abord et avant tout pour les besoins de la polémique européenne: "Toute la problématique du "despotisme oriental" est fallacieuse; elle a été fabriquée au xvIII siècle par les détracteurs de la monarchie française, comme un moyen d'attaquer cette forme de gouvernement » (p. 107)2 ${ }^{2}$. L'adéquation du système russe aux besoins de l'élite russe est une autre question. Sa fonctionnalité est certaine, mais le plus proche voisin de la Russie était la Pologne-Lituanie qui développa, dans un contexte socio-économique assez semblable, des institutions radicalement différentes où le pouvoir du roi était étroitement contrôlé. Cette adaptation n'était pas moins favorable aux intérêts de l'élite et la Rzeczpospolita du début du XVII ${ }^{e}$ siècle représentait un empire aussi vaste que la Russie...

\section{NOTES}

1. L'absence la plus regrettable est celle du livre de M. Mervaud et J.-C. Roberti, Une infinie brutalité : l'image de la Russie dans la France des XV et XVII siècles, Paris, 1991 (Cultures et sociétés de l'Est, 15). Citons aussi André Berelowitch, «La Moscovie dans l'Europe du XVII siècle : la marche vers l'Ouest (1618-1721) ", in Armée et diplomatie dans l'Europe du XVII siècle, Paris, 1992, p. 27-66 (Bulletin de l'Association des historiens modernistes des universités, 16) ; J. Jubé, La Religion, les mœurs et les usages des Moscovites, éd. par M. Mervaud, Oxford, 1992 (Studies on Voltaire and the 
eighteenth century, 294); J. Margeret, Un Mousquetaire à Moscou: mémoires sur la première révolution russe 1604-1614, éd. par A. Bennigsen, Paris, 1983; M. Mervaud, "Jacques Jubé et l'Union des Églises ", Revue des Études slaves, 70, 1998, p. 377-398 (numéro thématique intitulé : "La Chrétienté latine et les Slaves orientaux»). Giuseppe (et non Dzhuzeppe d'après une transcription du russe !) D'Amato n'a pas seulement publié Sočinenija ital'jancev o Rossii konca XVXVI vv., Moscou, 1995 (cité par Poe), mais aussi La Moscovia di fine '500 nei resoconti dei viaggiatori inglesi, Gênes, 1995 ; «Richard Chancellor alla scoperta della Moscovia », Europa Orientalis, 14, 1, 1995, p. 7-17 ; «Ital'jancy XVI veka o Rossii », in Rossija i Italija, fasc. 2, Moscou, 1995-1996, p. 34-49 ; "Gorod Moskva v vosprijatii ital'janskogo čitatelja XV-XVI vekov », Arheografičeskij ežegodnik, 1997, p. 103-107. Signalons enfin un récent recueil slovéno-russe que Poe semble ignorer: Žiga Herberstein odkritelj Rusije: XVI stoletje $v$ Rusiji in slovenskih deželah = Sigizmund Gerberštejn pervootkryvatel' Rossii : XVI stoletie v Rossii i slovenskih zemljah, Ljubljana, 1999.

2. L'auteur n'a pu non plus lire l'ouvrage, postérieur au sien, d'André Berelowitch, La hiérarchie des égaux: la noblesse russe d'Ancien Régime ( $\mathrm{XVI}^{\mathrm{e}}-\mathrm{XVII} \mathrm{I}^{\mathrm{e}}$ siècles), Paris, 2001 dont la première partie s'intitule Le despotisme paradoxal. Les p. 134 à 140, démontrant l'adhésion profonde d'une large couche de notables au système monarchique russe, sont très riches d'enseignement ( $c f$. mes comptes rendus dans la Revue des Études slaves, 72, 2000, p. 569-577 pour D. Ostrowski, 73, 2001, p. 540-542 pour A. Berelowitch). 